\title{
Higher Cord Blood Levels of Mannose-Binding Lectin-Associated Serine Protease-2 in Infants With Necrotising Enterocolitis
}

\author{
LUREGN J. SCHLAPBACH, CHRISTOPH AEBI, URS FISCH, ROLAND A. AMMANN, MARGRITH OTTH, SUSANNE BIGLER, \\ MATHIAS NELLE, STEFFEN BERGER, AND ULF KESSLER \\ Department of Pediatrics [L.J.S., C.A., U.F., R.A.A., M.O., M.N.], Department of Pediatric Surgery [S.B., U.K.], Institute for Infectious \\ Diseases [C.A., M.O.], University of Bern, 3010 Bern, Switzerland
}

\begin{abstract}
Necrotising enterocolitis (NEC) causes significant morbidity and mortality in premature infants. The role of innate immunity in the pathogenesis of NEC remains unclear. Mannosebinding lectin $(\mathrm{MBL})$ recognizes microorganisms and activates the complement system via MBL-associated serine protease-2 (MASP2). The aim of this study was to investigate whether MBL and MASP-2 are associated with NEC. This observational case-control study included 32 infants with radiologically confirmed NEC and 64 controls. MBL and MASP-2 were measured in cord blood using ELISA. Multivariate logistic regression was performed. Of the 32 NEC cases (median gestational age, $30.5 \mathrm{wk}$ ), 13 (41\%) were operated and $5(16 \%)$ died. MASP-2 cord blood concentration ranged from undetectable $(<10 \mathrm{ng} / \mathrm{mL})$ to $277 \mathrm{ng} / \mathrm{mL}$. Eighteen of $32(56 \%)$ NEC cases had higher MASP-2 levels $(\geq 30 \mathrm{ng} / \mathrm{mL})$ compared with 22 of $64(34 \%)$ controls (univariate OR 2.46; 95\% CI 1.03-5.85; $p=$ $0.043)$. Higher cord blood MASP-2 levels were significantly associated with an increased risk of NEC in multivariate analysis (OR 3.00; 95\% CI 1.17-7.93; $p=0.027)$. MBL levels were not associated with NEC $(p=0.64)$. In conclusion, infants later developing NEC had significantly higher MASP-2 cord blood levels compared with controls. Higher MASP-2 may favor complement-mediated inflammation and could thereby predispose to NEC. (Pediatr Res 64: $562-566,2008$ )
\end{abstract}

A lthough prognosis for premature infants has improved over the last years as a result of advances in perinatal and intensive care, morbidity and mortality due to necrotising enterocolitis (NEC) remain high (1). Numerous risk factors, including prematurity, intrauterine growth retardation, formula feeding, bacterial infection, and intestinal ischemia have been implicated in the pathogenesis of the disease $(2,3)$. However, since the exact pathophysiological mechanisms remain undefined, identification of infants at risk for NEC and early recognition of NEC represent a challenge (4).

Early infancy is a vulnerable period of transition from passive, transplacentally acquired immunity to individual, adaptive immunity. Since premature infants experience both humoral and cellular immunodeficiency, they rely on genetically determined innate defense mechanisms (5). Innate immunity is mainly mediated by host proteins that recognize conserved pathogen-associated molecular patterns, such as

Received March 4, 2008; accepted June 10, 2008

Correspondence: Ulf Kessler, M.D., Department of Pediatric Surgery, University of Bern, Inselspital, CH-3010 Bern, Switzerland; e-mail: ulf.kessler@insel.ch

Supported by a grant of the Rossi Research Fund, Department of Pediatrics, University of Bern, Switzerland. repetitive sugar arrays present on many microorganisms but not on mammalian cells (6). The lectin pathway of complement activation represents an important component of innate immunity and consists of Mannose-binding lectin (MBL) and $\mathrm{L}-$ and $\mathrm{H}$-ficolins, which recognize various microorganisms (6). Both MBL and ficolins rely on MBL-associated serine protease-2 (MASP-2) to activate the complement system (6). Upon binding of MBL/MASP-2 complexes to microbial surfaces, MASP-2 sequentially cleaves $\mathrm{C} 4$ and $\mathrm{C} 2$, thereby generating the $\mathrm{C} 3$ convertase $\mathrm{C} 4 \mathrm{bC} 2 \mathrm{~b}$, which finally leads to opsonization and lysis of pathogens and recruitment of inflammatory cells (7).

Due to single nucleotide polymorphisms within the MBL2 gene and the associated promotor region, MBL deficiency affects approximately $30 \%$ of the white population (6). MBL deficiency has been associated with neonatal sepsis and pneumonia $(8,9)$. Recently, a mutation has been identified in the MASP-2/MAp19 gene with a gene frequency of 1.3 to $6.3 \%$, resulting in decreased MASP-2 serum concentrations in heterozygous individuals (10). Although children with MASP-2 deficiency are prone to develop chemotherapy-related infections (11), the role of MASP-2 deficiency on susceptibility to infection in newborns remains unknown.

Since recent research indicates that the inflammatory reaction of the host may be crucial for the development of NEC $(2,3,12)$, we have hypothesized that the lectin pathway of complement activation may be involved in the pathogenesis of NEC. The aim of the present study was to investigate whether cord blood concentrations of MBL and MASP-2 are associated with later development of NEC.

\section{PATIENTS AND METHODS}

Infants born between April 2000 and August 2007 at the Department of Obstetrics and Gynecology, University of Bern, Switzerland, who developed NEC as defined by Bell stage II or III (13) were eligible for this study. Infants with major congenital malformations were excluded. The study was approved by the institutional review board including the fact that informed consent was not needed due to the retrospective nature of the study (Kantonale Ethikkommision, Direktion Forschung und Lehre, Inselspital, University of Bern, Bern, Switzerland). Treatment protocols of NEC and perinatal care of newborns did not substantially change during the study period.

\footnotetext{
Abbreviations: MASP-2, mannose-binding lectin-associated serine protease-2; MBL, mannose-binding lectin; NEC, necrotising enterocolitis; PDA, perstistent ductus arteriosus
} 
Controls and matching criteria. For each patient, two matched controls were searched using the institutional neonatal database. Controls were matched for the following criteria since these were considered the main potential confounders (2,3): 1) gestational age ( $\pm 1 \mathrm{wk})$; 2) small versus appropriate birth weight for gestational age (SGA: weight percentile $<10$ ); 3 ) prenatal maternal corticosteroid therapy for fetal lung maturation induction; 4) maternal chorioamnionitis, defined as maternal fever, elevated maternal C-reactive protein, fetal tachycardia, prolonged rupture of membranes and/or placental histology indicative of chorioamnionitis (14); and 5) perinatal asphyxia, defined as umbilical artery $\mathrm{pH}<7.10$, umbilical artery base excess $\leq-10$ and Apgar score at 5 min $<5$ (15). Infants were not eligible as controls if they had suffered from significant abdominal disease such as suspicion of NEC during hospital stay or if they underwent abdominal surgery for any disease.

Outcome measures and data assessment. Occurrence of NEC was defined as primary outcome. Secondary outcomes included death attributed to NEC, time between birth and development of NEC, NEC requiring surgery (either primary or after failed conservative therapy), and Bell stage III (13). Patient characteristics and clinical data were searched retrospectively using the institutional neonatal database and the patient charts.

MBL and MASP-2 measurements. Cord blood is routinely stored at our institution to determine Toxoplasma gondii serology in infants of mothers with unknown or negative serostatus. After centrifugation, cord blood serum was frozen in sterile tubes at $-20^{\circ} \mathrm{C}$. MBL and MASP- 2 concentrations were measured using a commercially available ELISA (MBL oligomer ELISA kit by Antibodyshop, Gentofte, Denmark; MASP-2 HK326 ELISA kit by HyCult biotechnology, Uden, The Netherlands). According to the manufacturer, the MASP-2 assay is validated for values as low as $1.6 \mathrm{ng} / \mathrm{mL}$. Due to the limited amount of cord blood serum available, a quantity of $40 \mathrm{uL}$ was used at a dilution of 1:6 resulting in a MASP-2 detection limit of $10 \mathrm{ng} / \mathrm{mL}$.

To compare the MASP-2 concentrations of this cohort with term neonates, blood was collected from 10 healthy term neonates at birth (cord blood) and at 1,3 , and 6 months.

Statistical analysis. Patients and controls were compared using uni- and multivariate logistic regression with NEC or severe NEC outcomes as dependent variable. The following variables were included as covariables in the multivariate model: all matching criteria (gestational age, intrauterine growth retardation, prenatal steroids, chorioamnionitis, perinatal asphyxia); variables that had shown a trend $(p<0.20)$ for association with NEC in univariate analyses (mode of delivery, birth weight); and previously described risk factors for NEC (sex, need for mechanical ventilation after birth, and persistent ductus arteriosus [PDA] requiring medical closure with indomethacine)
(16-19). Spearman's rank correlation, Mann-Whitney U-test and Wilcoxon's rank sum test were used where appropriate.

According to previous studies, low MBL levels were defined as MBL cord blood concentrations $<700 \mathrm{ng} / \mathrm{mL}(8,9)$. Contrary to MBL, no data exist on normal MASP-2 concentrations in neonates. Preliminary analysis showed that NEC patients had significantly more often MASP- 2 concentrations above detection limit $(10 \mathrm{ng} / \mathrm{mL})$ than controls (19 of 32 [59\%] versus 25 of 64 [39\%]; multivariate odds ratio [OR]2.66, 95\% confidence interval [CI]1.04$6.79, p=0.041)$. By means of receiver-operating-characteristics curve analysis, MASP-2 cord blood concentrations were categorized into lower MASP-2 levels $(<30 \mathrm{ng} / \mathrm{mL})$ and higher MASP-2 levels $(\geq 30 \mathrm{ng} / \mathrm{mL})$. Several sensitivity analyses were performed based on different cut-offs for MBL and MASP-2.

Two-sided tests were used throughout, and $p$-values below 0.05 were considered significant. SPSS 13.0 software was used for all analyses.

\section{RESULTS}

During the study period, 8819 infants were born at the Department of Obstetrics and Gynecology, University of Bern, and 48 infants developed NEC. Sixteen (33\%) children with NEC were excluded, because cord blood was unavailable. The remaining 32 infants with a median gestational age of 30.5 wk (range, 25-39) were enrolled as patients in the study. Baseline demographic data and presence of previously described risk factors for NEC were not significantly different between patients and controls (Table 1). Infants developed NEC at a median age of $11 \mathrm{~d}$ (range, 1-51, IQR 6 to 19). Thirteen $(41 \%)$ infants were operated and five $(16 \%)$ died during hospital treatment as a direct consequence of NEC.

In the whole study population (patients and controls), MASP-2 cord blood concentrations ranged from undetectable $(<10 \mathrm{ng} / \mathrm{mL})$ to $277 \mathrm{ng} / \mathrm{mL}$. Fifty-two of 96 (54\%) infants had MASP-2 concentrations below detection limit. MASP-2 concentration was neither associated with gestational age $(p=$

Table 1. Baseline characteristics and risk factors for NEC of patients and controls

\begin{tabular}{|c|c|c|c|}
\hline Characteristic & $\begin{array}{c}\text { Patients } n=32 \text { number } \\
\text { (IQR) or }(\%)\end{array}$ & $\begin{array}{l}\text { Controls } n=64 \text { number } \\
\text { (IQR) or }(\%)\end{array}$ & $p^{*}$ \\
\hline Male sex & $13(40 \%)$ & $31(48 \%)$ & 0.47 \\
\hline Gestational age $\dagger[\mathrm{wk}]$ & $31(27-33)$ & $31(28-33)$ & 0.43 \\
\hline Birth weight $[\mathrm{g}]$ & $1185(794-1795)$ & $1452(1032-1891)$ & 0.19 \\
\hline Birth weight percentile & $20(9-45)$ & $22(7-50)$ & 0.44 \\
\hline $\mathrm{SGA} \dagger$ & $11(34 \%)$ & $22(34 \%)$ & 1.00 \\
\hline Prenatal steroids $\dagger$ & $25(78 \%)$ & $50(78 \%)$ & 1.00 \\
\hline Maternal chorioamnionitis $\dagger$ & $10(31 \%)$ & $20(31 \%)$ & 1.00 \\
\hline Perinatal Asphyxia $\dagger$ & $4(13 \%)$ & $8(13 \%)$ & 1.00 \\
\hline Maternal fever & $2(6 \%)$ & $5(8 \%)$ & 0.78 \\
\hline PROM & $5(16 \%)$ & $7(11 \%)$ & 0.52 \\
\hline Cesarean section & $25(78 \%)$ & $40(63 \%)$ & 0.13 \\
\hline Apgar $1 \mathrm{~min}$ & $6(4-7)$ & $7(4-8)$ & 0.26 \\
\hline Apgar 5 min & $8(7-9)$ & $8(7-9)$ & 0.65 \\
\hline Apgar $10 \mathrm{~min}$ & $8(8-9)$ & $9(8-9)$ & 0.31 \\
\hline Umbilical artery $\mathrm{pH}$ & $7.28(7.22-7.33)$ & $7.30(7.25-7.33)$ & 0.34 \\
\hline Umbilical artery base excess & $-2.0(-0.6$ to -6.2$)$ & $-2.2(-0.3$ to -4.1$)$ & 0.36 \\
\hline Mechanical ventilation $\ddagger$ & $15(47 \%)$ & $22(34 \%)$ & 0.24 \\
\hline Duration of ventilation $\neq[\mathrm{d}]$ & $0(0-2)$ & $0(0-1)$ & 0.99 \\
\hline Antibiotic treatment $\neq$ & $14(44 \%)$ & $29(45 \%)$ & 0.89 \\
\hline PDA & $5(16 \%)$ & $8(13 \%)$ & 0.67 \\
\hline
\end{tabular}

Median values and (interquartile ranges, IQR), or number and (percentage) are given where appropriate.

PDA, persistent ductus arteriosus; PROM, prolonged rupture of membranes; SGA, small for gestational age.

$* p$-value of univariate logistic regression.

$\dagger$ Matching criteria.

$\$$ Before development of necrotizing enterocolitis. 
0.933 ) nor with any of the clinical parameters studied. MBL cord blood concentrations ranged from 29 to $4104 \mathrm{ng} / \mathrm{mL}$ with a median of $863 \mathrm{ng} / \mathrm{mL}$ and were positively correlated with gestational age and birth weight $(p=0.024$ and 0.014 , respectively). The duration of storage did not significantly affect MBL or MASP-2 concentration (visual assessment and Spearman's rank correlation; data not shown) (20).

Median MASP-2 cord blood concentration was higher in NEC cases than in controls (median $38 \mathrm{ng} / \mathrm{mL}$ versus $<10$ $\mathrm{ng} / \mathrm{mL})$. Eighteen of $32(56 \%)$ NEC cases versus 22 of 64 (34\%) controls had higher MASP-2 cord blood levels $(\geq 30$ $\mathrm{ng} / \mathrm{mL}$; univariate OR $2.46,95 \% \mathrm{CI}, 1.03-5.85, p=0.043$ ) (Fig. 1). Multivariate analysis adjusted for all matching criteria, sex, birth weight, mode of delivery, need for mechanical ventilation, and PDA, confirmed that infants developing NEC had significantly more often higher MASP-2 levels compared with controls (OR, 3.00; 95\% CI, 1.17-7.93; $p=0.027$ ).

Sixteen of $32(50 \%)$ NEC cases versus 26 of $64(41 \%)$ controls had low MBL cord blood levels. No significant difference was found when comparing MBL levels between patients and controls (univariate OR, 1.46; 95\% CI, 0.62-3.43; $p=0.384$; multivariate $\mathrm{OR}, 1.25 ; 95 \% \mathrm{CI}, 0.49-3.22 ; p=$ 0.644). Several sensitivity analyses based on different cut-offs for MBL and MASP-2 were performed which confirmed the main results (data not shown).

None of the severe NEC outcomes, e.g. death, need for surgery or advanced Bell stage, was significantly associated with MBL or MASP-2 levels (data not shown). Infants with higher MASP-2 levels tended to develop NEC earlier than infants with lower MASP-2 levels (median 10.5 versus $15 \mathrm{~d}$; $p=0.323$ ).

To explore the course of MASP-2 during infancy, we measured MASP-2 in 10 healthy term newborns in cord blood and over the first 6 mo of life. MASP- 2 concentrations increased significantly during the first 6 mo (median at birth 128 $\mathrm{ng} / \mathrm{mL}$, at $6 \mathrm{mo} 418 \mathrm{ng} / \mathrm{mL} ; p=0.043$ ), with the main rise occurring during the first 3 mo $(p=0.018)$, see Figure 2 . MASP-2 cord blood concentrations in these term infants were significantly higher compared with premature infants $(p=$ 0.002).

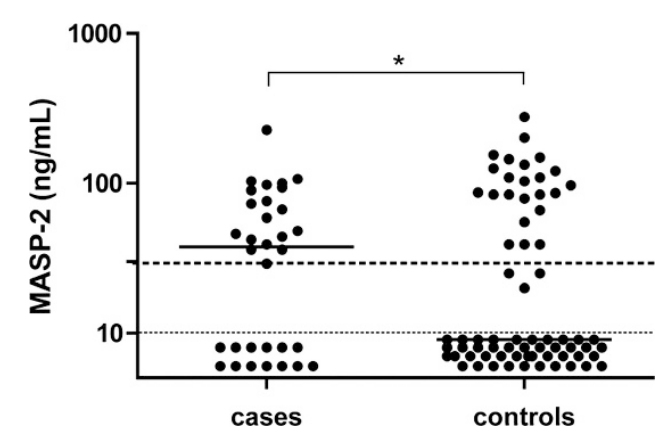

Figure 1. Comparison of MASP-2 cord blood concentrations between patients $(n=32)$ and controls $(n=64)$. The horizontal dashed line is set at $30 \mathrm{ng} / \mathrm{mL}$ indicating lower and higher MASP-2 levels. The horizontal dotted line is set at $10 \mathrm{ng} / \mathrm{mL}$ indicating detection limit. $* p<0.05$.

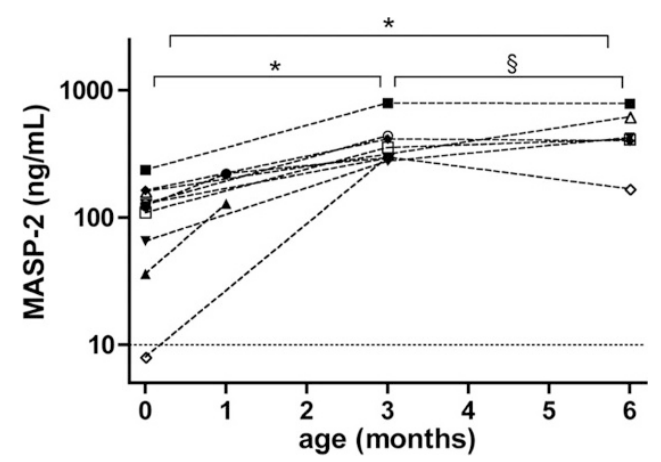

Figure 2. Age-dependent rise of MASP-2 concentrations during the first six months of live in ten healthy term neonates. The horizontal dotted line is set at $10 \mathrm{ng} / \mathrm{mL}$ indicating detection limit. $* p<0.05 ; \S p>0.05$.

\section{DISCUSSION}

The results of this case-control study indicate that higher MASP-2 cord blood levels are associated with a 3-fold increased risk to develop NEC. To the best of our knowledge, this is the first study investigating the role of the lectin pathway of complement activation in NEC.

Clinical studies on MASP-2 have reported an increased susceptibility to infection in MASP-2 deficient patients $(11,21)$, whereas elevated MASP-2 levels were associated with a worse prognosis in adults with colorectal cancer, possibly by contributing to tumor-associated inflammation (22). Recent studies have shown that MBL can aggravate reperfusion injury (24) and that the lectin pathway of complement activation can increase injury after intestinal ischemia (25). Since MASP-2 represents the common pathway for both MBL and ficolins (7), higher MASP-2 levels in neonates may enhance complement activation after initial triggers, thereby generating potentially harmful inflammatory mediators such as C3a and C5a (26) and could thereby promote the development of NEC. The observed trend for infants with higher MASP-2 to develop NEC earlier than infants with lower MASP-2 is in line with this concept. Even if innate immunity plays a key role in the protection against invading organisms and in the establishment of the gut flora, its implication in NEC is poorly understood $(3,27)$. The pathophysiological concept of NEC postulates that initially hypoperfusion and gastrointestinal immaturity lead to an increased vulnerability of the intestinal tract facilitating bacterial invasion $(2,3)$. Research on the role of platelet activating factor and $\mathrm{NF} \kappa \mathrm{B}$ in NEC models has demonstrated that the reaction of the host is decisive on whether NEC develops then $(2,3,12)$.

Overall MASP-2 cord blood concentrations in most neonates in our study were extremely low in comparison to values reported from adults and children $(7,11,20,22)$. In a subset of healthy term infants, we were able to show that MASP-2 concentrations rise significantly during infancy approaching adult levels at the age of 6 mo (20). Our results thus suggest that MASP-2 deficiency during the neonatal period is physiologic and that the lectin pathway of complement activation is not fully functional at birth in most premature infants. This novel finding has implications on the research on associated proteins, since both MBL and Ficolins rely on MASP-2 to 
activate complement. The finding of higher cord blood MASP-2 in infants later developing NEC in contrast to the extremely low MASP-2 concentrations found in most premature neonates suggests that MASP-2 deficiency may represent a protective mechanism against excessive proinflammatory stimuli during the neonatal period.

No association was found between cord blood MBL and NEC. The concentrations of MBL measured in our cohort were in good agreement with published results and correlated with gestational age, as reported previously $(8,9)$. Several authors have reported increased frequency of neonatal sepsis and pneumonia in MBL deficient neonates $(8,9)$, whereas two studies which were based on MBL genotype could not confirm this finding $(28,29)$. The protective role of MBL and MASP-2 found in studies focusing on sepsis, which is usually caused by a single pathogen, apparently does not apply to multifactorial NEC.

The strengths of this study include the detailed matching aimed at minimizing potential confounders $(3,16,17)$. A relevant bias due to acute-phase reactions in cord blood measurements is unlikely, since we carefully matched infants for perinatal factors that may affect the hepatic synthesis of MBL and MASP-2. Comparison of nonmatched perinatal characteristics revealed no significant differences between patients and controls. Since data on normal MASP-2 concentrations in the neonatal period are lacking, receiver-operating-characteristics curve analysis was used to define MASP-2 levels. Although we have not determined MASP-2 concentrations during NEC, we assume that cord blood concentrations influence MASP-2 levels during the first weeks of life when NEC usually develops, similar to MBL (8,31). In addition, determining MASP-2 in cord blood has the advantage of obtaining baseline values that are independent from acute-phase reactions associated with NEC itself.

This study has some limitations, mostly related to its retrospective design. Thirty-three percent of infants who developed NEC during the study period were excluded because cord blood serum was unavailable. A selection bias, however, seems unlikely, since the clinical characteristics of the patients included are in good agreement with a larger NEC cohort recently described (4). Similar to most studies on NEC (3,32-34), sample size is a major limitation when interpreting results particularly with regard to secondary NEC outcomes.

In conclusion, we report that higher cord blood MASP-2 levels were significantly associated with a 3-fold increased risk to develop NEC. Although independent replication by other cohorts is needed, our results suggest that higher MASP-2 may represent a previously unrecognized risk factor for NEC. Whether determination of MASP-2 may help to identify infants at increased risk of NEC and to improve early recognition of NEC needs to be assessed in future prospective studies. In contrast, the novel finding of extremely low MASP-2 concentrations in most premature infants compared with the values reported from older children suggests that the lectin pathway of complement activation is often not fully functional at birth. Further studies should therefore address the role and maturation of the lectin pathway of complement activation, including ficolins, in the neonatal period.
Acknowledgments. The authors thank Lorenz Risch, M.D., Risch Laboratories, Bern, for providing us with serum samples of healthy term neonates, and Tina Brehmer, Department of Pediatric Surgery, University of Bern, for help in data acquisition.

\section{REFERENCES}

1. Fanaroff AA, Stoll BJ, Wright LL, Carlo WA, Ehrenkranz RA, Stark AR, Bauer CR, Donovan EF, Korones SB, Laptook AR, Lemons JA, Oh W, Papile LA, Shankaran S, Stevenson DK, Tyson JE, Poole WK; NICHD Neonatal Research Network 2007 Trends in neonatal morbidity and mortality for very low birthweight infants. Am J Obstet Gynecol 196:147.e1-e8

2. Caplan MS, Simon D, Jilling T 2005 The role of PAF, TLR, and the inflammatory response in neonatal necrotizing enterocolitis. Semin Pediatr Surg 14:145-151

3. Lin PW, Stoll BJ 2006 Necrotising enterocolitis. Lancet 368:1271-1283

4. Kessler U, Mungnirandr A, Nelle M, Nimmo AF, Zachariou Z, Berger S 2006 A simple presurgical necrotizing enterocolitis-mortality scoring system. J Perinatol 26:764-768

5. Strunk T, Richmond P, Simmer K, Currie A, Levy O, Burgner D 2007 Neonatal immune responses to coagulase-negative staphylococci. Curr Opin Infect Dis 20:370-375

6. Thiel S 2007 Complement activating soluble pattern recognition molecules with collagen-like regions, mannan-binding lectin, ficolins, and associated proteins. Mol Immunol 44:3875-3888

7. Sorensen R, Thiel S, Jensenius JC 2005 Mannan-binding-lectin-associated serine proteases, characteristics and disease associations. Springer Semin Immunopathol 27:299-319

8. Frakking FN, Brouwer N, van Eijkelenburg NK, Merkus MP, Kuijpers TW, Offringa M, Dolman KM 2007 Low mannose-binding lectin (MBL) levels in neonates with pneumonia and sepsis. Clin Exp Immunol 150:255-262

9. de Benedetti F, Auriti C, D'Urbano LE, Ronchetti MP, Rava L, Tozzi A, Ugazio AG, Orzalesi MM 2007 Low serum levels of mannose binding lectin are a risk factor for neonatal sepsis. Pediatr Res 61:325-328

10. Stengaard-Pedersen K, Thiel S, Gadjeva M, Moller-Kristensen M, Sorensen R, Jensen LT, Sjoholm AG, Fugger L, Jensenius JC 2003 Inherited deficiency of mannan-binding lectin-associated serine protease 2. N Engl J Med 349:554-560

11. Schlapbach LJ, Aebi C, Otth M, Leibundgut K, Hirt A, Ammann RA 2007 Deficiency of mannose-binding lectin-associated serine protease- 2 associated with increased risk of fever and neutropenia in pediatric cancer patients. Pediatr Infect Dis J 26:989-994

12. Le Mandat Schultz A, Bonnard A, Barreau F, Aigrain Y, Pierre-Louis C, Berrebi D, Peuchmaur M 2007 Expression of TLR-2, TLR-4, NOD2 and pNF-kappaB in a Neonatal Rat Model of Necrotizing Enterocolitis. PLoS One 2:e1102

13. Bell MJ, Ternberg JL, Feigin RD, Keating JP, Marshall R, Barton L, Brotherton T 1978 Neonatal necrotizing enterocolitis. Therapeutic decisions based upon clinical staging. Ann Surg 187:1-7

14. Kaukola T, Herva R, Perhomaa M, Paakko E, Kingsmore S, Vainionpaa L, Hallman M 2006 Population cohort associating chorioamnionitis, cord inflammatory cytokines and neurologic outcome in very preterm, extremely low birth weight infants. Pediatr Res 59:478-483

15. Jacobs S, Hunt R, Tarnow-Mordi W, Inder T, Davis P 2007 Cooling for newborns with hypoxic ischaemic encephalopathy. Cochrane Database Syst Rev CD003311

16. Sankaran K, Puckett B, Lee DS, Seshia M, Boulton J, Qiu Z, Lee SK 2004 Variations in incidence of necrotizing enterocolitis in Canadian neonatal intensive care units. J Pediatr Gastroenterol Nutr 39:366-372

17. Llanos AR, Moss ME, Pinzon MC, Dye T, Sinkin RA, Kendig JW 2002 Epidemiology of neonatal necrotising enterocolitis: a population-based study. Paediatr Perinat Epidemiol 16:342-349

18. Herrera C, Holberton J, Davis P 2007 Prolonged versus short course of indomethacin for the treatment of patent ductus arteriosus in preterm infants. Cochrane Database Syst Rev CD003480

19. Holman RC, Stoll BJ, Clarke MJ, Glass RI 1997 The epidemiology of necrotizing enterocolitis infant mortality in the United States. Am J Public Health 87:2026-2031

20. Moller-Kristensen M, Jensenius JC, Jensen L, Thielens N, Rossi V, Arlaud G, Thiel S 2003 Levels of mannan-binding lectin-associated serine protease- 2 in healthy individuals. J Immunol Methods 282:159-167

21. Olesen HV, Jensenius JC, Steffensen R, Thiel S, Schiotz PO 2006 The mannanbinding lectin pathway and lung disease in cystic fibrosis-dysfunction of mannanbinding lectin-associated serine protease 2 (MASP-2) may be a major modifier. Clin Immunol 121:324-331

22. Ytting H, Christensen IJ, Thiel S, Jensenius JC, Nielsen HJ 2005 Serum mannanbinding lectin-associated serine protease 2 levels in colorectal cancer: relation to recurrence and mortality. Clin Cancer Res 11:1441-1446

23. Deleted in proof

24. Walsh MC, Bourcier T, Takahashi K, Shi L, Busche MN, Rother RP, Solomon SD, Ezekowitz RA, Stahl GL 2005 Mannose-binding lectin is a regulator of inflammation that accompanies myocardial ischemia and reperfusion injury. J Immunol 175:541-546

25. Hart ML, Ceonzo KA, Shaffer LA, Takahashi K, Rother RP, Reenstra WR, Buras JA, Stahl GL 2005 Gastrointestinal ischemia-reperfusion injury is lectin complement pathway dependent without involving C1q. J Immunol 174:6373-6380 
26. Guo RF, Ward PA 2005 Role of C5a in inflammatory responses. Annu Rev Immunol 23:821-852

27. Neu J, Chen M, Beierle E 2005 Intestinal innate immunity: how does it relate to the pathogenesis of necrotizing enterocolitis. Semin Pediatr Surg 14:137-144

28. van der Zwet WC, Catsburg A, van Elburg RM, Savelkoul PH, VandenbrouckeGrauls CM 2008 Mannose-binding lectin (MBL) genotype in relation to risk of nosocomial infection in pre-term neonates in the neonatal intensive care unit. Clin Microbiol Infect 14:130-135

29. Ahrens P, Kattner E, Kohler B, Hartel C, Seidenberg J, Segerer H, Moller J, Gopel W 2004 Mutations of genes involved in the innate immune system as predictors of sepsis in very low birth weight infants. Pediatr Res 55:652-656

30. Deleted in proof
31. Aittoniemi J, Miettinen A, Laippala P, Isolauri E, Viikari J, Ruuska T, Soppi E 1996 Age-dependent variation in the serum concentration of mannan-binding protein. Acta Paediatr 85:906-909

32. Moonen RM, Paulussen AD, Souren NY, Kessels AG, Rubio-Gozalbo ME, Villamor E 2007 Carbamoyl phosphate synthetase polymorphisms as a risk factor for necrotizing enterocolitis. Pediatr Res 62:188-190

33. Henderson G, Craig S, Baier J, Helps N, Brocklehurst P, McGuire W 2007 Cytokine gene polymorphisms in preterm infants with necrotising enterocolitis: genetic association study. Arch Dis Child Fetal Neonatal Ed, in press

34. Szebeni B, Szekeres R, Rusai K, Vannay A, Veres G, Treszl A, Arato A, Tulassay T, Vasarhelyi B 2006 Genetic polymorphisms of CD14, toll-like receptor 4, and caspase-recruitment domain 15 are not associated with necrotizing enterocolitis in very low birth weight infants. J Pediatr Gastroenterol Nutr 42:27-31 